\title{
[EN-A-35] Macroscopic Analysis to Identify Stage Boundaries in Multi-Stage Arrival Management
}

\author{
(EIWAC 2019) \\ ${ }^{+}$E. Itoh*, Y. Miyazawa*, M. Finke**, J. Rataj** \\ *ATM Department \\ Electronic Navigation Research Institute (ENRI), \\ National Institute of Maritime, Port and Aviation Technology (MPAT) \\ Tokyo, Japan \\ [eri |miyazawa_y]@mpat.go.jp \\ **Institute of Flight Guidance \\ Department of Controller Assistance \\ German Aerospace Center (DLR) \\ Braunschweig, Germany \\ [michael.finke | juergen.rataj]@dlr.de
}

\begin{abstract}
Accommodating the air traffic growth, reducing arrival delay is one of the most important functions of designing the ATM system. One of the newest concepts to further optimize arrival flows is multi-stage arrival management, proposed by DLR, in which different guidance principles to manage the arriving traffic are implemented in different stages. These stages are optimized to the core management task to be done in a certain area of the arrival stream and the conditions of the surrounding environment. This paper discusses this concept through a macroscopic analysis on the overall arrival traffic flows. Further, this paper analyzes parts of the multi-stage arrival management concept applied to Tokyo International Airport as a case study. A stochastic characteristic of arrival trajectories will be discussed as a counterpart of conventional deterministic trajectorybased operation based on data-driven analysis and arrival procedures at the airport. The best strategies of shifting arrival flow control to time-based management is analyzed based on the stochastic data analysis. Impacts of pop-up aircraft are discussed as one of the causes to increase uncertainties in aircraft trajectory management.
\end{abstract}

Keywords: Arrival Management, Multi-Stage, Arrival Delay, Air Traffic Management

\section{INTRODUCTION}

Highly frequented airports are the critical elements in air traffic. In the most cases they are directly or implicitly the origin of delays in the air traffic system. Therefore, arrival management is an essential part in air traffic management. To clarify the meaning of arrival management the following definition from EUROCONTROL[1] is used: "Arrival Management is a general term given to the process of safely and effectively arranging arrivals into a smooth efficient flow for landing at a destination airport". To improve the arrival management process, controllers (ATCo) are supported by a family of decision support systems called arrival managers (AMANs). According to [1], an AMAN is defined as a software specifically designed to provide assistance in metering and sequencing arrival streams and that delivers information needed to implement an efficient arrival management. Following this definition, the first AMANs were built already more than 20 years ago. The tasks where the controller is usually supported by an AMAN are:
- Build an arrival sequence.

- Assign an arrival time at the runway threshold and other significant waypoints for each aircraft in the sequence.

- Predict a trajectory for each aircraft which implements the assigned landing time.

- Transform the trajectory into appropriate guidance instructions which are transmitted to the pilot.

Since the first AMANs became operational, a steady development takes place to increase their abilities, their accuracy and their performance to support the controller because of the high impact of these systems for the whole air traffic. The last development in arrival management in the Single European Sky Air Traffic Management Research Initiative (SESAR) addresses the extension of the planning horizon of an AMAN from approx. 80NM to more than $350 \mathrm{NM}$ [2]. With this extension, delay absorbing maneuvers in the terminal area like holdings and arrival transitions shall be shifted to speed reductions in higher flight levels during cruise flight. This approach shall reduce 
fuel consumption and environmental impact as well as controller workload in the TMA due to the shorter stay in lower altitudes. Although the benefits of extended AMAN are already indicated[3] this approach will nevertheless not solve all problems. Due to the very limited speed range within which an aircraft can safely be flown at cruising level, the extension of the AMAN horizon has to be very big to create delays comparable to conventional holdings. For example, when considering an Airbus A330 with a minimum cruise speed of Mach 0.79 and its maximum cruise speed of Mach 0.86, the extended AMAN horizon has to have a radius of at least $420 \mathrm{NM}$ to compensate a delay equivalent to a standard holding pattern of 4 minutes [4]. Furthermore, increasing the capacity on an airport using a re-categorization of wake turbulence separations creates new challenges for the arrival management, but this time, close to the runway. Hence, a more holistic concept concerning arrival management and its controller assistance functions is necessary.

\section{DRAWBACKS OF MODERN ARRIVAL MANAGEMENT SYSTEMS}

One drawback of current AMAN designs is the effect of model uncertainties in the trajectory calculation and the probability of disturbances. As a consequence, the individual trajectory reliability decreases considerably with increasing distance of the aircraft to the airport. Hence, the drawback of the extension of the current planning horizon from $80 \mathrm{NM}$ by a factor of 5 is the decreasing predictability and accuracy of the calculated trajectory. Furthermore, horizons of 500 nautical miles and more causes that some aircraft in the approach flow are still on the ground, as they are departing from airports that are less than 500 nautical miles away from the destination. Because the uncertainties as well as the probability of disturbances for a flight on ground are much higher as it is the case for airborne flights, the predictability and the quality of the trajectory are even worse. Hence, without additional effort concerning the concept of operation and the used methods as well as the existing planning mechanisms, the new approach results in decreased prediction quality of arrival time and increased number of arrival sequence changes with negative effects on the aircraft, ATC and airport operation. Moreover, corrective measures for an individual aircraft far away from the airport may become useless due to unexpected new disturbances that again reduce efficiency.

Following the recently introduced new trajectory based operation (TBO) concepts, the constraint to stay on a preplanned but unprecise trajectory results in an increased number of control actions for the aircraft. Negative consequences of increased control actions is again an increased fuel consumption and, more important, an increased maintenance effort for the engines as well as a low acceptance by the pilots due to their increased workload.
The described drawbacks require the development of new concepts of operation, methods and planning algorithms for AMANs, taking the drawbacks into account, which stem from uncertainties and disturbances. Different solutions for airborne arrivals and arrivals that are still on ground at their departure aerodrome are needed as the different status has a significant influence on the uncertainties as well as the probability of disturbances impacting a flight. Hence, using todays planning and guidance functionalities for arrival management is disadvantageous.

The future arrival management will further be strongly influenced by the introduction of new wake turbulence categories. In the past a $3 * 3$ matrix was used to define the separation distances between the subsequent aircraft. These aircraft categories are heavy, medium and light and the number of different separations in the $3 * 3$ matrix is 3 ( $4 \mathrm{NM}$, $5 \mathrm{NM}, 6 \mathrm{NM})[5]$. In this case, the controllers are able to remember this low amount of numbers of different separation minima for the aircraft pairs, hence no further support was required to apply them to the aircraft on final approach. With the introduction of RECAT-EU, as an example, the number of aircraft categories increases to 6 and the number of different separation values to 8 in a $6^{*} 6$ matrix [6]. The new wake turbulence categories increase the complexity for the controller considerably. To deal with that the controller needs additional support tools to create an efficient arrival sequence. Further considerations go in the direction to assign every individual aircraft pair an individual separation [7]. To guide such a separation scheme it is indispensable to intensively support the controller with technical means.

\section{CONCEPT OF MULTI-STAGE ARRIVAL MANAGEMENT}

Following the description in the previous chapters, it becomes apparent that more and more tasks of the arrival management are collected under an AMAN, which makes these systems more and more complex. By extending the planning horizon considerably, arrival management starts much earlier than in the past. The implementation of new separation values requires new support elements in the AMAN close to the runway.

It can be concluded, that the driving requirements, rules to be followed and constraints to be considered in future arrival management will at least depend on

- the distance of the airplane to the destination aerodrome,

- the characteristics and local constraints of main arrival flows,

- the diversity of involved aircraft type performance and equipment,

- the flight phase, 


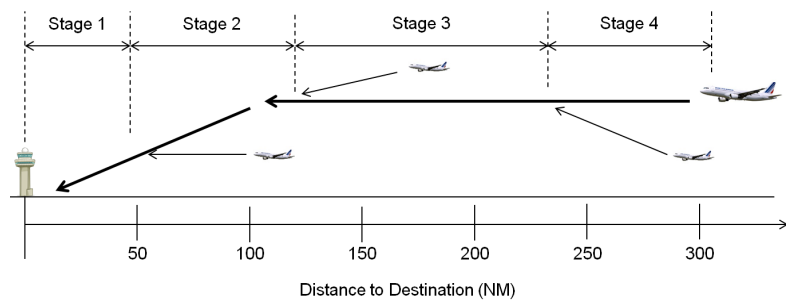

Figure 1 Fictional Example of a Possible Multi-Stage AMAN Design

- predictability of traffic as well as accuracy and uncertainty of planning systems.

Although the list of dependencies can surely be further extended, state of the art AMANs are still using the same algorithms for the whole traffic up to the planning horizon while not or not sufficiently considering the items contained in the list above. In addition, especially trajectory based deterministic calculations seem to be unsuitable without a minimum level of predictability and only tolerate minor uncertainties. A better solution could be to adapt and optimize the working principles of AMAN systems according to these dependencies taking into account system architecture considerations.

This would result in a "clustering" of coherent tasks, methods or procedures, following the "separation of concerns" software architecture principle [8]. Supplementary to "separation of concerns" is the "single responsibility principle" in the software engineering domain. Taking these software architecture principles into account, it is necessary to divide future AMANs with their various "concerns" in different modules.

This approach can be realized with a Multi-Stage AMAN design defined by DLR, where several different subsystems can use different working principles on different flight phases, arrival flows or distances from the destination aerodrome. This design allows using the working principle which fits best to the single stages and offers also a lot of optimization potential for the whole AMAN architecture at the individual airports (see Figure 1).

At its current stage of development, the Multi-Stage AMAN concept includes the following types of stages already:

- classical 4D-trajectory-based, fully deterministic stages,

- time-based stages

- flow-based stages

- stages using stochastic methods.

Further types of AMAN stages may be available in the future.

In addition, inspired by electric circuit or space launcher construction principles, two different types of stage arrangements can be identified:
- $\quad$ serial arrangement, where one stage directly feeds the traffic into another stage,

- parallel arrangement, where separate airspaces, arrival flows or otherwise clustered arrivals are planned and handled by two stages in parallel.

Based on the description of the preceding chapters a first stage/concern could be defined for the final approach area of an airport, where the main task is to create a tight sequence of aircraft on the center line by minimizing the used buffers on top of the minimum wake turbulence separation. At hub airports this task is done by the so called "Director" controller position which is very much specialized and focused just on this single task of creating the safe and effective sequence of aircraft on final track. Furthermore, the increasing task complexity by new wake turbulence categories and the focus on a time dependent separation between subsequent aircraft increases the distance of concerns to a trajectory based approach.

A second stage/concern could be driven by trajectory based operation with the goal to create an optimized aircraft sequence and feeding of the first stage defined above. Optimized aircraft sequence means, that the order of the arriving aircraft corresponds to a previously defined optimization criterion. Furthermore, a high quality concerning the trajectory calculation is necessary to enable continuous descent approaches with engines in idle which are especially environment friendly. Here, it is important to start the calculation of the aircraft trajectory at least before the top of descent. Additional to sequencing, the trajectory based approach enables a collision detection and avoidance in the TMA which is of utmost importance to ensure a safe and orderly traffic flow.

A third stage could be allocated to the concern of arrival management of distant flights still in the en-route phase of flight. Following the draw backs in chapter 2 another approach to guide the traffic should be taken into account here. This approach bases on statistical information which enables a continuous flow of aircraft to the airport without a high amount of corrective action. In contrast to this approach, precise trajectory based operations would require a high amount of corrective actions of the aircraft as they have to follow their precisely planned but uncertain trajectories. More details concerning the statistical arrival management approach will be presented in an own paper in the near future.

In order to build an AMAN system according to the multistage design, several decisions have to be made:

- type of the stage used for a defined part of the whole arrival management,

- serial or parallel arrangement of the individual stages,

- data exchange and transition conditions between the different stages, 


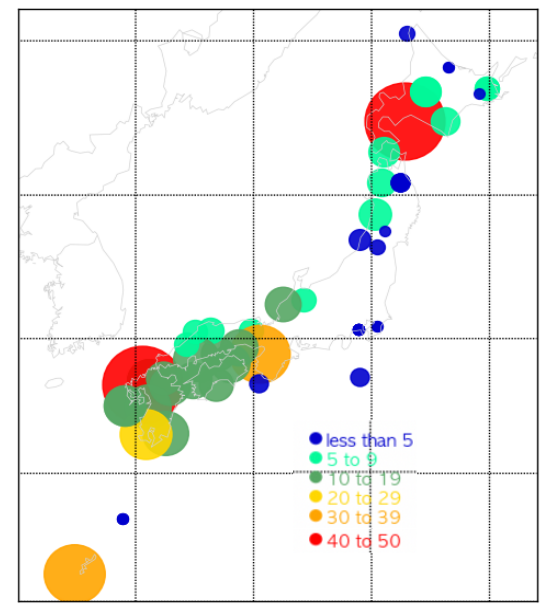

Figure 2 Distributions of departure airports with average number of departures arriving at Tokyo International Airport in a day

- definite borders between the different stages / concerns.

A first scientific study has already been made by the Japanese research Electronic Navigation Research Institute (ENRI) on the definition of a possible border between flow based and trajectory based arrival management for TokyoHaneda International Airport, which is described in the following chapters.

\section{DATA-DRIVEN ANALYSIS}

\subsection{Case study data description - Tokyo International Airport}

Prior to discuss stage boundaries in the arrival management, this section introduces aircraft arrival operations at Tokyo International Airport, which this paper focuses on as a case study airport.

A total, maximum number of 447,000 departures and arrivals are accepted per year, with a maximum 80 operations in one hour. The airport makes use of four runways on daily basis, while the choice of the runway configuration depends on wind direction.

Over $60 \%$ of the domestic flights in Japan are concentrated at this airport. Figure 2 shows the distribution of departure airports with average number of departure aircraft arriving at Tokyo International Airport in Japan.

\subsection{Stochastic Analysis on Air Traffic Arrival Flow}

In order to characterize arrival traffic flow in each stage (see Fig.1), a data-driven analysis is conducted using two-years of radar tracks and flight plans in 2016 and 2017. Figure 3 shows definitions of aircraft flight time and inter-arrival time using concentric circles centered at Tokyo International Airport. In total, 29 data sets of aircraft flight

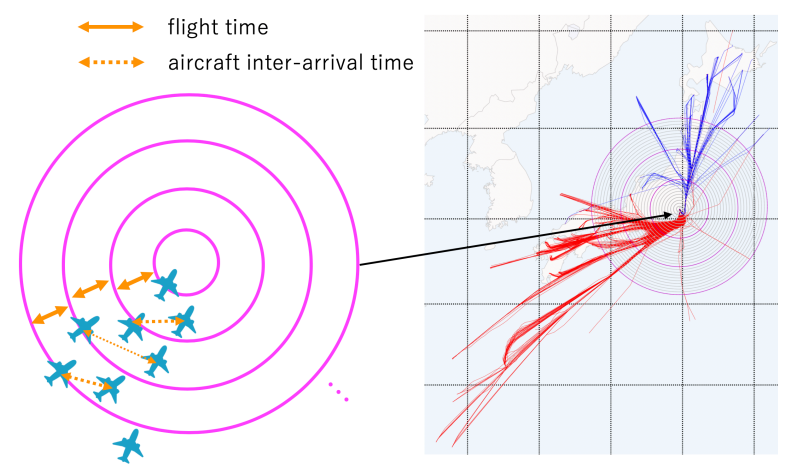

Figure 3 Definitions of flight time and aircraft inter-arrival time in this paper. Map of Japan is drawn with flight tracks during an entire day in November 2016 [9]. The red tracks show the south-west traffic flow. The blue tracks show the north traffic flow.

time and inter-arrival time are defined when using a maximum radius of $300 \mathrm{NM}$ with an increment of $10 \mathrm{NM}$. Aircraft inter-arrival time is defined as aircraft time-spacing between preceding and succeeding aircraft at each concentric circle. For example, the first data set is difference of aircraft's crossing time after its proceeding aircraft crossed at the circle with $20 \mathrm{NM}$ radius, the second data set is the time difference at the circle with $30 \mathrm{NM}$ radius, and so on.

Authors' past works indicated that the empirical distributions of the aircraft flight time could be numerically approximated using Gaussian distribution [9][10]. The significant features of arrival traffic control appeared in the variances of the aircraft flight time distributions. Figure 4 compares flight time distributions corresponding to the arrival traffic flows from south-west direction. One of the most significant arrival strategies is illustrated in Fig.4 for airspace between concentric circles $30 \mathrm{NM}$ and $40 \mathrm{NM}$, $40 \mathrm{NM}$ and $50 \mathrm{NM}$ radii, where the variances grows dramatically in the arrival traffic flows from south-west direction. This explained by the fact that the arrival timespacing was actively conducted by the air traffic controllers in the airspace between 30NM and 50NM away from the airport, just before the aircraft enter the terminal area. The increase in both the mean flight time and the flight time variance in the direction of the airport is due to the airspeed reduction that arriving aircraft undertake prior to landing. For flight time, the mean and variance converge close to the circle of radius 200NM. This circle captures current arrival strategies, since this is the airspace within which the traffic control capacity is met and the spacing at merging points is filled. Between circles of radii 200NM and 300NM, air traffic controllers make an effort to maintain safe and efficient traffic flows by prioritizing airlines' own procedures. In summary, there are three main strategies illustrated in Fig. 4: (i) arrival time-spacing within the circle around 50NM, especially between the 30 and $40 \mathrm{NM}$ radii circles; (ii) arrival metering for traffic capacity control and spacing at merging points between the 50 and $200 \mathrm{NM}$ 


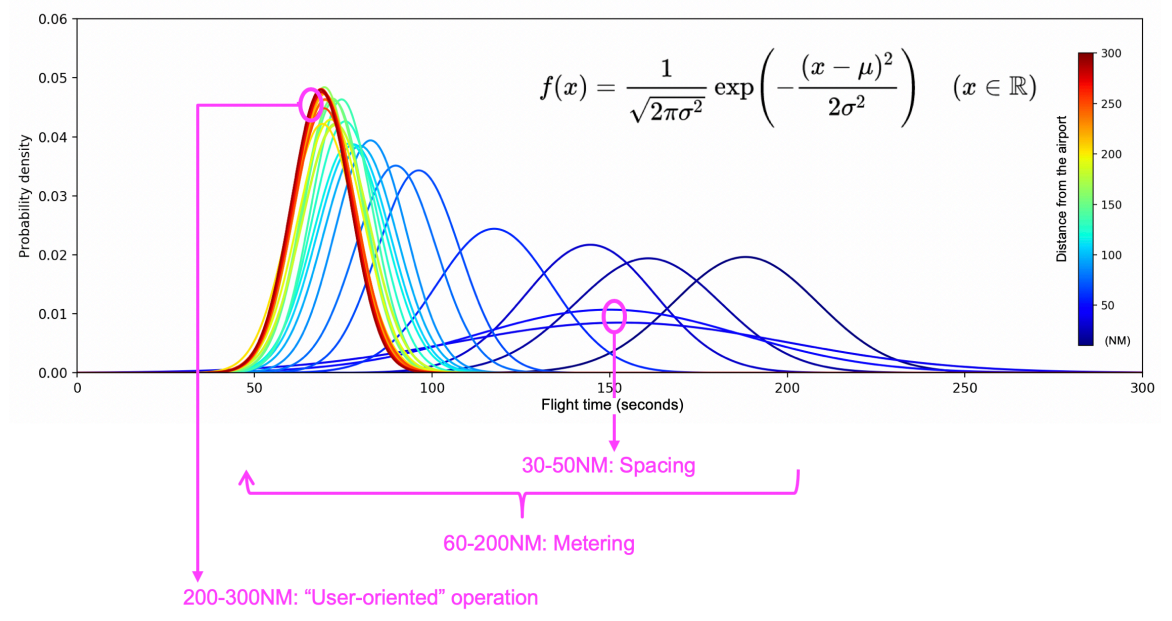

Figure 4 Comparing flight time distributions every 10NM radius, 10NM to $300 \mathrm{NM}$

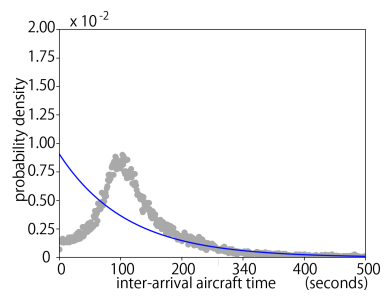

(1) $50 \mathrm{NM}, C_{e}=0.4688$

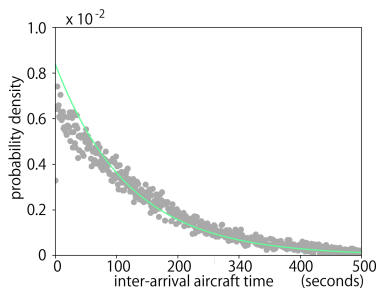

(3) $150 \mathrm{NM}, C_{e}=0.8089$

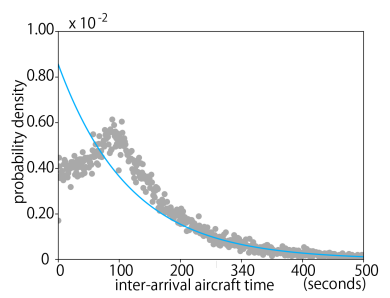

(2) $100 \mathrm{NM}, C_{e}=0.6467$

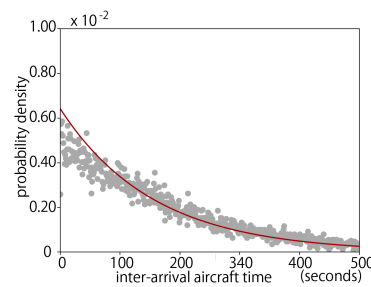

(4) $300 \mathrm{NM}, C_{e}=0.8125$

Figure 5 Empirical distribution of the aircraft inter-arrival time and exponential fittings [10][11]

circles; and (iii) maintaining efficient traffic flow by prioritizing airlines' own procedures beyond the 200NM circle. Minimizing arrival delays and operational costs requires great consideration in combining these different strategies.

Figure 5 compares exponential fittings and empirical probability densities of the aircraft inter-arrival time from the south-west direction at concentric circles with 50NM, $100 \mathrm{NM}, 150 \mathrm{NM}$, and 300NM radii [10]. The empirical distribution of the inter-arrival time is well approximated by an exponential distribution where the arriving aircraft fly further than the circle around 150NM radius. However, the inter-arrival times converge to a nearly Gaussian distribution towards the arrival airports.

The empirical coefficient is defined as $C_{e}=\sqrt{\sigma_{A}} / \mathbb{E}[A]$, where $\mathbb{E}[A]$ and $\sigma_{A}$ are the mean inter-arrival time and variance of the inter-arrival time. When $C_{e} \rightarrow 1$, the empirical distribution of the inter-arrival time is well approximated by an exponential distribution. If $C_{e}$ is much larger or smaller than 1, the empirical distribution is deviating from the exponential distribution. According to the empirical data, $C_{e}=0.4688,0.6467,0.8089,0.8125$ corresponding to $50,100,150$, and $300 \mathrm{NM}$ radii of concentric circles.

\section{EXPLORING CONTROL STRATEGIES IN FUTURE ARRIVAL MANAGEMENT}

\subsection{Shifting Air Traffic Control Flow to Time-Based Traffic Management}

One of the important arrival strategies to determine is where and how the aircraft arrival flow shifts to time management towards the arrival airport while minimizing arrival delay time. The first author's study clarified the best arrival strategy based on the analysis applying data-driven queuing models [10][11][12]. The proposed queue-based approach demonstrated that there are five parameters, which impact on arrival delay time as follows: 1) arrival traffic rate 2) airspace capacity 3) mean of aircraft flight time 4) variance of aircraft flight time 5) variance of aircraft inter-arrival time. All five parameters were estimated through datadriven analysis using the two years radar tracks and flight plans. Mean and variance of aircraft flight time, and variance of aircraft inter-arrival time was analyzed in Section 4.2 as shown in Figs. 4 and 5.

Figure 6 summarizes current arrival traffic rate in an hour at each concentric circle with $10 \mathrm{NM}$ to $300 \mathrm{NM}$ radii every $10 \mathrm{NM}$, focusing on arrival traffic from the north and southwest direction during the most congested time 


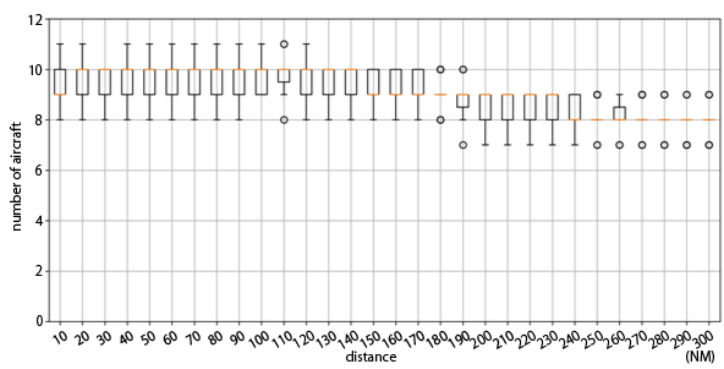

(1) Arrival traffic from the north direction, 17:00-22:00

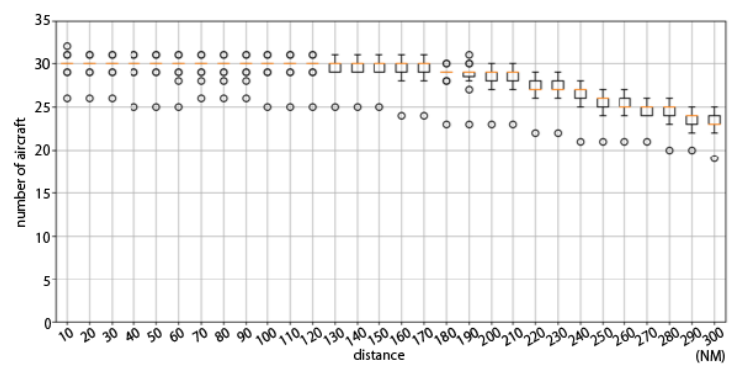

(2) Arrival traffic from the south-west direction, 17:00-22:00

Figure 6 Arrival rate in an hour [12]

period 17:00 to 22:00 based on the data statistics. Arrival rate is well-controlled at each airspace during the most congested time period; approximately 10 aircraft from the north (see Fig.6(1)) and 30 aircraft (see Fig.6(2)) from the south-west arrives within 150NM radius concentric circle in an hour [12].

Table 1 counted the number of aircraft from the south-west direction, which flew in the defined airspace, every 10 minutes during 17:00 to 22:00 time period in the two years. The values are relevant from operational point of views; $7 \mathrm{NM}$ separation was given to arrival aircraft at $30 \mathrm{NM}$ to $40 \mathrm{NM}$ away from the airport before entering terminal area, thus the number of aircraft between 30NM and 40NM radii concentric circles was 1 (see median value at $30-40 \mathrm{NM}$ in Table 1.) Approximately 5NM separation was given to Initial Approach Fix, so the median took 2 aircraft in 1020NM and 20-30NM airspace (see Table 3). In the queueing model, these values directory define the capacity at the assigned airspace.

Based on the proposed queue-based approaches $[10][11][12]$, the best arrival strategy to minimize arrival time delay is increasing airspace capacity within around 50NM radius range. Extending aircraft flight time (with delay time) is more effective where the variances of interarrival time is smaller, thus absorbing aircraft arrival delay closer to the airport is relevant operation for mitigating arrival delay time in the current arrival operation.
Table 1 Arrival aircraft numbers served in the assigned airspace

\begin{tabular}{|c|c|c|c|c|c|c|c|c|c|c|}
\hline $\begin{array}{l}\text { Airspace } \\
\text { (NM) }\end{array}$ & $\begin{array}{l}10^{-} \\
20\end{array}$ & $\begin{array}{l}20^{-} \\
30\end{array}$ & $\begin{array}{l}30^{-} \\
40\end{array}$ & $\begin{array}{l}40^{-} \\
50\end{array}$ & $\begin{array}{l}50^{-} \\
60\end{array}$ & $\begin{array}{l}60^{-} \\
70\end{array}$ & $\begin{array}{l}70^{-} \\
80\end{array}$ & $\begin{array}{l}80^{-} \\
90\end{array}$ & $\begin{array}{l}90^{-} \\
100\end{array}$ & $\begin{array}{l}100^{-} \\
110\end{array}$ \\
\hline median & 2 & 2 & 1 & 2 & 1 & 1 & 1 & 1 & 1 & 1 \\
\hline mean & 1.79 & 1.85 & 1.38 & 1.66 & 1.52 & 1.10 & 0.908 & 0.875 & 0.812 & 0.819 \\
\hline STD & 0.809 & 0.971 & 0.649 & 0.882 & 0.914 & 0.808 & 0.753 & 0.746 & 0.758 & 0.793 \\
\hline $\begin{array}{l}\text { Airspace } \\
\text { (NM) }\end{array}$ & $\begin{array}{l}110^{-} \\
120\end{array}$ & $\begin{array}{l}120^{-} \\
130\end{array}$ & $\begin{array}{l}130^{-} \\
140\end{array}$ & $\begin{array}{l}140^{-} \\
150\end{array}$ & $\begin{array}{l}150^{-} \\
160\end{array}$ & $\begin{array}{l}160^{-} \\
170\end{array}$ & $\begin{array}{l}170^{-} \\
180\end{array}$ & $\begin{array}{l}180^{-} \\
190\end{array}$ & $\begin{array}{l}190^{-} \\
200\end{array}$ & $\begin{array}{l}200^{-} \\
210\end{array}$ \\
\hline median & 1 & 1 & 1 & 1 & 1 & 0 & 1 & 0 & 0 & 0 \\
\hline mean & 0.773 & 0.792 & 0.723 & 0.693 & 0.676 & 0.647 & 0.683 & 0.585 & 0.593 & 0.581 \\
\hline STD & 0.801 & 0.861 & 0.790 & 0.802 & 0.794 & 0.779 & 0.806 & 0.745 & 0.734 & 0.771 \\
\hline $\begin{array}{l}\text { Airspace } \\
\text { (NM) }\end{array}$ & $\begin{array}{l}210^{-} \\
220\end{array}$ & $\begin{array}{l}220^{-} \\
230\end{array}$ & $\begin{array}{l}230^{-} \\
240\end{array}$ & $\begin{array}{l}240^{-} \\
250\end{array}$ & $\begin{array}{l}250^{-} \\
260\end{array}$ & $\begin{array}{l}260^{-} \\
270\end{array}$ & $\begin{array}{l}270^{-} \\
280\end{array}$ & $\begin{array}{l}280^{-} \\
290\end{array}$ & $\begin{array}{l}290^{-} \\
300\end{array}$ & \\
\hline median & 1 & 0 & 0 & 0 & 0 & 0 & 0 & 0 & 0 & \\
\hline mean & 0.807 & 0.555 & 0.654 & 0.627 & 0.542 & 0.603 & 0.518 & 0.626 & 0.536 & \\
\hline STD & 0.837 & 0.715 & 0.804 & 0.783 & 0.744 & 0.755 & 0.699 & 0.739 & 0.709 & \\
\hline
\end{tabular}

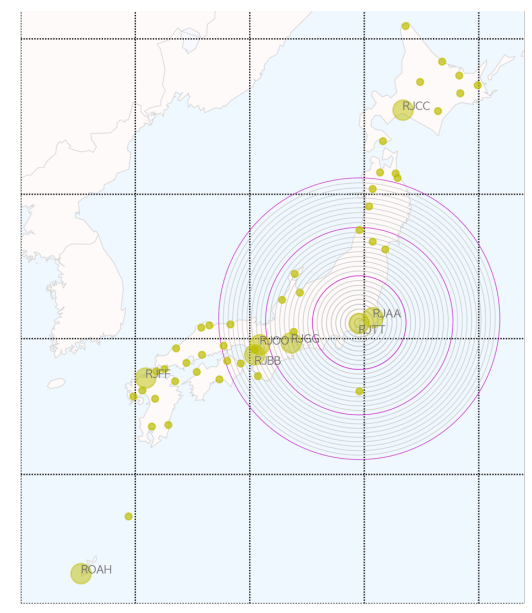

Figure 7 Distributions of airports in Japan

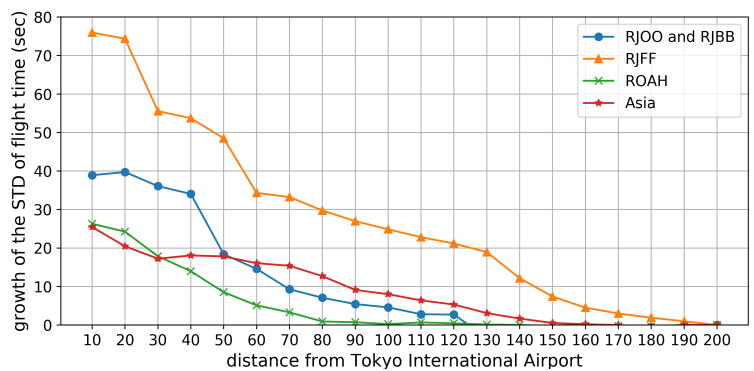

Figure 8 Comparing growth of the flight time STD from these at $200 \mathrm{NM}$

\subsection{Uncertainties in Time Management due to Pop-up Aircraft}

Although smaller variances of aircraft flight time and interarrival time mitigates arrival delay time under limited airspace capacities, uncertainties in aircraft time management increases in further airspace from arrival airport. One of the causes anticipated is the impact of popup aircraft departing within the considered horizon from the arrival airport, which merge into air traffic flow. As shown in Figure 7, Osaka International Airport (RJOO) and 


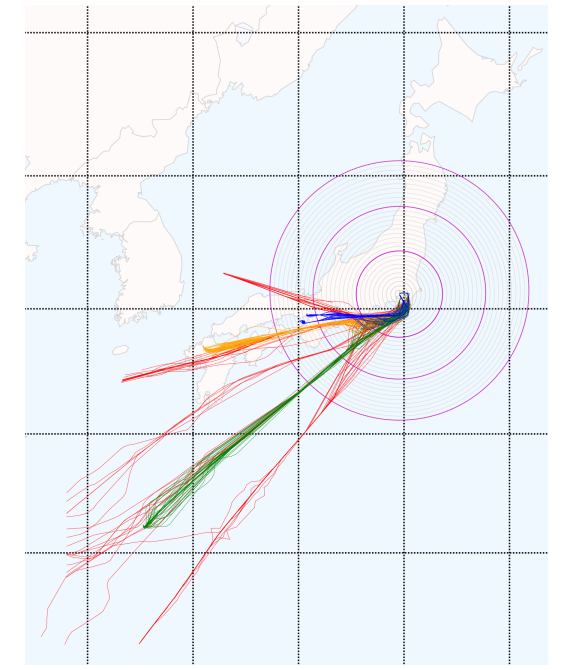

(1) Horizontal records

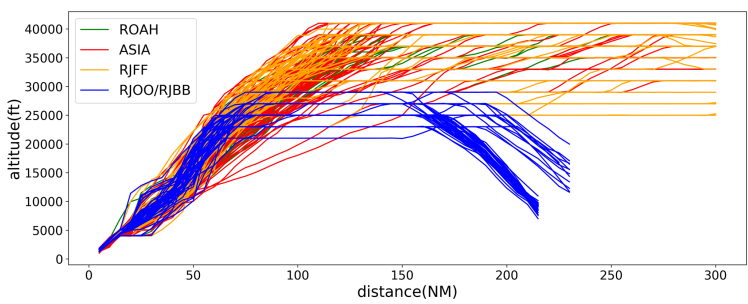

(2) Vertical records

Figure 9 Flight tracks of arrival aircraft from RJFF, RJOO, RJBB, ROAH, and Asian countries in a day

Kansai International Airport (RJBB) locates between Fukuoka International Airport (RJFF) and Tokyo International Airport. Figure 3 also indicates that arrivals from these airports take a large amount in total amount of arrivals at Tokyo International Airport.

In order to analyze the impact of pop-up aircraft departing from RJOO and RJBB, Figure 8 compares growth of standard deviation (STD) in flight time since the aircraft departing from RJFF, RJOO, RJBB, Naha International Airport (ROAH), and Asian countries crossed the 200NM radius circle centered at Tokyo International Airport. Figure 10 compares the horizontal and vertical track records in a day. Although variances of flight time impacted by the total amount of flight, Fig.8 shows that the STD corresponding to arrivals from RJFF grows between $130 \mathrm{NM}$ and $160 \mathrm{NM}$, where departing flights from RJOO and RJBB merge into traffic departing from RJFF. As shown in Fig. 9, flight tracks of RJFF, RJOO, and RJBB merges not only horizontally, but also vertically below $30,000 \mathrm{ft}$ airspace.

\section{DISCUSSION}

This section discusses efficient arrival management strategies which can be applied in the stages of a multi-stage AMAN architecture (see Fig.1) proposed in Section 3 based

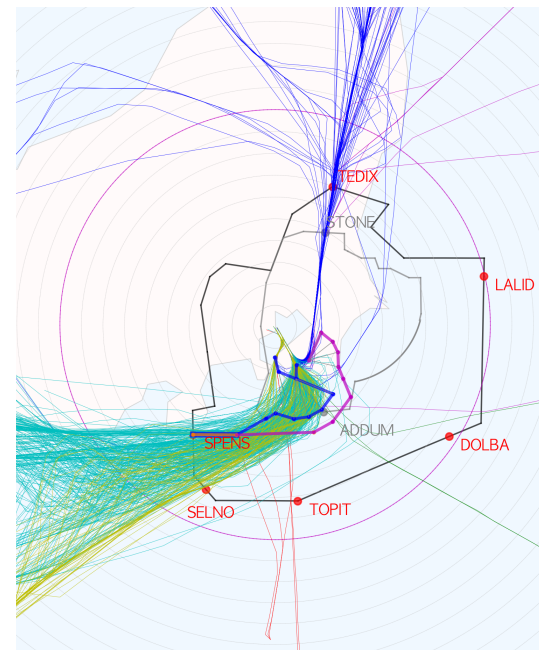

Figure 10 One of the representative point-merge operation at Tokyo International Airport

on the data-driven analysis in section 4 and 5, and the first authors' work in ref. [10][11][12].

Firstly, the best arrival strategy in the stage 1 is increasing airspace capacity, especially 30NM-50NM distance from Tokyo International Airport for minimizing arrival delay time. This control would be done by achieving timemanagement of aircraft inter-arrival time at around 70NM from the arrival airport in the stage 2, suggested in ref.[10]. Freeze horizon, where the AMAN calculates the optimal arrival schedules, is required to lie in the stage 3 prior to achieve arrival scheduling. Aircraft trajectories would be controlled between the freeze horizon and horizon where time management is required to achieve.

Secondly, total aircraft arrival delay time is reduced if the flight time is extended in the airspace area where the variance of aircraft inter-arrival time is smaller. As shown in Fig. 5, the variance of the inter-aircraft time grows in the farther airspace area from the arrival airport, and the distribution is near exponential at the airspaces farther than $150 \mathrm{NM}$. One of our future challenges is to propose the best way to draw horizons, which tailor arrival aircraft traffic prior to the conventional freeze horizon, in stage 3 and/or stage 4.

Thirdly, again it is repeated but controlling aircraft flight time, which increases the variance of aircraft flight time in arrival traffic flow, is allowed where the variances of the inter-aircraft time is smaller. Otherwise, arrival delay time of the total arrival traffic increases. This also means that trajectory optimization targeting individual aircraft is not the solution to achieve minimum aircraft arrival delay because of the interference with surrounding traffic. Trajectory-based operation need to be designed in order to reduce the variance of inter-arrival times in the arrival traffic. 
Fourthly, pop-up aircraft also impacts on increasing the variances of aircraft flight time. Further study will analyze the impacts on the arrival traffic delay and solutions to mitigate the impacts.

Lastly, applying new wake turbulence minima, so called RECAT (Wake Turbulence Re-categorization), and pointmerge operation influences in the stage 1. Point-merge operation is introduced in arrival traffic at Tokyo International Airport since July 2019. Figure 10 shows an example of point-merge routes applying to arrivals from south-west direction, with flight tracks of arrivals in a day in 2017 and concentric circles drawn every 10 NM radius. There were two transition points named ADDUM and STONE previously, however six transition points are newly prepared according to the expansion of terminal area. As shown in Fig.10, the arcs of point-merge routes locate within 50NM radii concentric circle. Authors' future work will also analyze the impacts of the RECAT and pointmerge operations on the arrival aircraft traffic.

\section{CONCLUSIONS}

This paper introduced a multi-stage arrival management concept and discussed stage boundaries based on a datadriven analysis. Two years of data consisting of radar tracks and flight plans of air traffic arriving at Tokyo International Airport were analyzed as a case study. The analytical results characterized arrival traffic flow at each stage in the proposed concept. The best arrival strategy was discussed to shift air traffic flow control to time management. Authors' future works further discuss the efficient design of arrival management in each stage.

Design requirements of arrival management system depend on the characteristics of a given arrival air traffic flow and its surrounding environment, e. g., runway and airspace capacity, weather conditions, air routes, and other geographical constraints. This study further designing the multi-stage arrival management not only at Tokyo International Airport, but also different airports, e. g. German airports, and conduct interoperability analysis in the future.

\section{ACKNOWLEDGMENTS}

This research was conducted under CARATS initiatives supported by the Civil Aviation Bureau, Ministry of Land, Infrastructure, Transport and Tourism (MLIT) as the "Studies on the Extended Arrival Management". This research was also supported by the Ministry of Education, Culture, Sports, Science and Technology (MEXT) as the "Post-K Computer Exploratory Challenge" (Project ID: hp180188).

\section{REFERENCES}

[1] EUROCONTROL, "Arrival Manager Implementation Guidelines and Lessons learned" Edition 0.1, December 2010. p.14.

[2] SESAR Joint Undertaking, "xStream Demonstration Plan", Edition 00.02.00, May 2018, not published

[3] SESAR Joint Undertaking, "iStream Demonstration Report", Edition 00.02.00, November 2016

[4] Modern Airliners, http://www.modernairliners.com/airbus-a330/airbusa330-specs/, accessed July 2019

[5] International Civil Aviation Organization (ICAO), "Procedures for Air Navigation Services - Air Traffic Management", Doc 4444, 15th Edition, 2007, p.4-11.

[6] EUROCONTROL, "RECAT-EU - European Wake Turbulence Categorisation and Separation Minima on Approach and Departure", Edition 1.1, July 2015

[7] https://ext.eurocontrol.int/lexicon/index.php/Pairwise_separation, accessed July 2019

[8] Effektive Softwarearchitekturen: Ein praktischer Leitfaden, Carl Hanser Verlag GmbH \& Co. KG, ISBN-13: 978-3446452077

[9] E. Itoh and Y. Miyazawa, "A Study on the Stochastic Modeling of Traffic Flows Arriving to Tokyo International Airport", Proceedings of The $56^{\text {th }}$ Aircraft Symposium (written in Japanese), 2018.

[10] E. Itoh and M. Mitici, "Queue-based Modeling of the Aircraft Arrival Process at a Single Airport", Submitted to Topical Collection "Air TransportationOperations and Management", Aerospace, 6(10), 103; 2019.

[11] E. Itoh and M. Mitici, "Analyzing Tactical Control Strategies for Aircraft Arrivals at an Airport Using Queue-based Modeling", Submitted to Journal of Air Transport Management (Under Review), 2019.

[12] E. Itoh an M. Mitici, "Evaluating the Impact of New Aircraft Separation Minima on Available Airspace Capacity and Arrival Time Delay", Submitted to The Aeronautical Journal (Accepted), 2019.

\section{Copyright Statement}

The authors confirm that they, and/or their company or institution, hold copyright of all original material included in their paper. They also confirm they have obtained permission, from the copyright holder of any third party material included in their paper, to publish it as part of their paper. The authors grant full permission for the publication and distribution of their paper as part of the EIWAC2019 proceedings or as individual off-prints from the proceeding. 